\title{
EFFICIENT NUMERICAL COMPUTATION OF SECOND-HARMONIC SCATTERING BY CLUSTERS OF NANOPARTICLES
}

\author{
Ivan Sekulic*(1) and Nicolae C. Panoiu ${ }^{(1)}$ \\ (1) University College London, Torrington Place, London WC1E 7JE, United Kingdom; e-mail: \\ i.sekulic@ucl.ac.uk; n.panoiu@ucl.ac.uk
}

In this paper, we introduce a transfer matrix method (TMM) for efficient calculation of second-harmonic generation (SHG) from clusters of nanospheres made of centrosymmetric materials. Different from current TMM formulations, our approach goes beyond the single particle configuration, thus greatly expanding its practical relevance. In addition, we incorporate in our method the contributions of both the surface and bulk nonlinear polarizations. This is especially valuable for the accurate analysis of purely dielectric systems, such as clusters of silicon or silica nanospheres, which are widely used in nanophotonics.

We proceed with constructing our numerical method in three steps [1]: first, we apply the TMM to calculate the total fields at the fundamental frequency (FF), which is the excitation frequency, then we use these computed fields inside the nanospheres to construct the nonlinear polarization sources, which play the role of excitation sources at the second harmonic (SH). Finally, the TMM is invoked again, and the SH fields and cross-sections of interest are evaluated. The linear TMM part of the algorithm is constructed in a well-known manner, namely starting with the surface integral representation of Maxwell equations, which maps the unknown equivalent surface electric and magnetic currents to the known excitation field. The unknown equivalent currents, dyadic Green's function and the known incident field oscillating at the FF are expanded in a basis set of vector spherical wave functions (VSWFs). A single-sphere transfer matrix, relating the incident to the scattered field expansion coefficients is obtained using the linear tangential field boundary conditions. Finally, in the construction of the transfer matrix of the cluster at the FF, the coupling between the spheres is considered by exploiting the translational formulae for VSWFs at the FF.

The nonlinear part of the TMM takes as the input the expansion coefficients of the linear internal fields linked to each particle which are used to expand the nonlinear SH polarization sources into a series of VSWFs. Similarly to the approach used in the linear regime, we expand the scattered and the internal SH electromagnetic fields in a series of VSWFs. The SH transfer matrix pertinent to each nanosphere, relating scattered to the excitation SH field expansion coefficients, is constructed by invoking nonlinear tangential boundary conditions at the surface of each nanosphere. The multiparticle transfer matrix is then obtained by considering multiple SH scattering among the spheres with the help of translational relations computed at the SH frequency.

The proposed numerical method is faster and has lower memory requirements when compared to the wellestablished numerical techniques, such as the finite-element method (FEM) and boundary-element method (BEM). This convenient feature stems from the fact that the entries of the transfer matrix related to each sphere, both at FF and $\mathrm{SH}$, can be computed analytically and are equal to the well-known linear/nonlinear Mie coefficients. Moreover, the cluster transfer matrix renders itself to efficient parallel implementation on highperformance computing platforms. As an example of how our method can be used, we present in Fig. 1. an analysis of a finite cubic array of nanospheres illuminated by a plane wave. The FEM analysis of such a cluster is prohibitive due to the memory resources associated with the discretization of a large computational domain. On the other hand, our method converges rapidly, using only a few hundreds of basis functions per sphere.
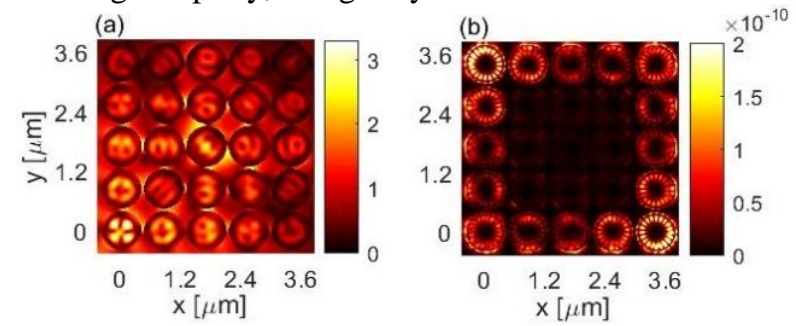

Figure 1. (a), (b) Field profiles at the FF and SH, respectively, determined for a 3D array of 125 silicon spheres.

1. I. Sekulic, J. W. You, and N. C. Panoiu, "T-matrix method for calculation of second-harmonic generation in clusters of spherical particles”, J. Quant. Spectrosc. Radiat. Transfer, vol. 268, 107643, 2021. 